\title{
Données nouvelles sur le métabolisme des principaux éléments-traces chez le dromadaire
}

\author{
B. Faye ${ }^{1^{*}}$ M. Bengoumi ${ }^{2}$
}

\begin{abstract}
Mots-clés
Dromadaire - Cuivre - Zinc - Fer Manganèse - Oligoélément Métabolisme des minéraux Complément alimentaire - M aroc.
\end{abstract}

\begin{abstract}
Résumé
Dans un essai effectué sur cinq dromadaires recevant une ration de base couvrant leurs besoins d'entretien, une complémentation minérale à base de sulfates de cuivre, zinc, manganèse et cobalt, d'iodure et de sélénite a été distribuée pendant trois mois, après trois semaines d'adaptation. Après arrêt de la complémentation, les animaux ont été suivis pendant trois autres mois. Des prélèvements de sang, de fèces et de foie par biopsie ont été régulièrement effectués pour doser le cuivre et le zinc. Le fer et le manganèse ont été analysés dans les fèces et les rations seulement. Au cours des trois phases de l'essai, la cuprémie moyenne était de 44,63 et $57 \mu \mathrm{g} / 100 \mathrm{ml}$ et la zincémie de 35,36 et $42 \mu \mathrm{g} / 100 \mathrm{ml}$. La complémentation minérale a donc augmenté les concentrations en cuivre au niveau plasmatique et hépatique, mais n'a pas modifié les teneurs en zinc. La concentration fécale de cuivre, zinc et manganèse a augmenté pendant la phase de complémentation, mais le taux d'absorption a globalement diminué de 15 à 20 p. 100, sauf celui du zinc qui est resté stable. La concentration du fer fécal a augmenté pendant la phase de complémentation en dépit de l'absence d'apport ferrique, indiquant une interaction négative entre minéraux mineurs et fer. L'ensemble des résultats permet de considérer les besoins du dromadaire en éléments-traces comme étant plus faibles que pour les autres ruminants.
\end{abstract}

De nombreux travaux récents ont permis de préciser en quoi consistait, sur le plan physiologique, l'adaptation légendaire du dromadaire aux conditions d'élevage extensif en milieu aride. Notamment, la régulation endocrinienne du métabolisme hydrominéral a pu être précisée $(9,21)$ ainsi que les mécanismes d'adaptation à la restriction hydrique (5). Concernant les éléments-traces, les études relatives au dromadaire sont demeurées éparses (11) et essentiellement descriptives $(7,8)$. Pourtant, les variations physiologiques observées et les réponses à la complémentation minérale (14) laissaient suggérer un métabolisme particulier du dromadaire, par comparaison avec les autres espèces de ruminants domestiques.

\footnotetext{
1. CIRAD-EMVT, Campus international de Baillarguet, BP 5035, 34032 Montpellier Cedex 1, France

2. Département de biochimie clinique et nutritionnelle, Institut Agronomique et Vétérinaire Hassan II, BP 6202, Rabat Instituts, Maroc

* Adresse pour la correspondance
}

L'essentiel des données disponibles dans la littérature est centré sur les valeurs observées des concentrations plasmatiques ou sériques $(1,12)$. Bien que ces valeurs constituent des indicateurs du statut nutritionnel en éléments-traces, elles ne renseignent pas sur le métabolisme de ceux-ci. Aussi la présente publication expose les données acquises récemment sur la régulation en élémentstraces dans l'espèce caméline et évalue ainsi les relations entre l'ingestion, le stockage hépatique et l'excrétion fécale. Bien entendu, la méthode factorielle classique utilisée pour déterminer le coefficient d'absorption réelle (CAR) n'est pas applicable dans le cas des éléments-traces, les besoins nets n'étant pas bien connus (17). Cette étude a été limitée aux éléments principaux dont le dosage est relativement aisé et les références disponibles dans la littérature : cuivre, zinc, manganèse et fer.

\section{MATERIEL ET METHODES}

L'essai a été réalisé à la station expérimentale du Gharb de l'Institut Agronomique et Vétérinaire Hassan II, Maroc. 


\section{Les animaux}

Cinq dromadaires âgés de 5 à 7 ans et en provenance du sud marocain ont été utilisés. Ils s'agissait de femelles taries non-gestantes. Leur poids était approximativement de $400 \mathrm{~kg}$. Elles étaient traitées contre les parasitoses externes et internes avec de l'ivermectine (Ivomec ND). Les animaux sont restés en bonne santé pendant toute la durée de l'expérience. Simultanément, cinq vaches taries de race Pie-noire ont été utilisées dans le même essai. Elles recevaient la même ration alimentaire et étaient soumises au même protocole de prélèvements. Cependant, seuls les résultats concernant les dromadaires ont été exposés ici, ceux relatifs aux bovins n'intervenant que dans la discussion.

\section{Procédure expérimentale}

Pendant toute la durée de l'essai, les animaux ont été maintenus dans des box individuels. Ils recevaient une ration de base comprenant $3 \mathrm{~kg}$ de paille de blé, $1,5 \mathrm{~kg}$ de farine de riz et $1,5 \mathrm{~kg}$ de mélasse. Cette ration était distribuée individuellement. Il n'y eut aucun refus au cours de l'expérimentation. Cette ration était censée couvrir globalement les besoins d'entretien des animaux. La distribution d'eau de boisson était ad libitum. Après une période d'adaptation au régime de base pour conférer aux cinq animaux un statut nutritionnel comparable, la période expérimentale (195 jours) a comporté 3 phases :

1. Une période de contrôle (J1-J21) au cours de laquelle les animaux ne recevaient que la ration de base sans aucune adjonction d'éléments minéraux.

2. Une période de supplémentation minérale (J22-J112) au cours de laquelle a été préparé quotidiennement un complément minéral distribué individuellement. Ce complément contenait, pour l'ensemble des dix animaux, 9,5 $\mathrm{g}$ de sulfate de cuivre, $44 \mathrm{~g}$ de sulfate de zinc, 30,1 g de sulfate de manganèse, $153,5 \mathrm{mg}$ d'iodure de calcium, 95,2 mg de sulfate de cobalt et 43,6 mg de sélénite de sodium, ce qui correspondait à un apport individuel quotidien de $240 \mathrm{mg}$ de cuivre, $1 \mathrm{~g}$ de zinc, $1 \mathrm{~g}$ de manganèse, $10 \mathrm{mg}$ d'iode, $2 \mathrm{mg}$ de cobalt et $2 \mathrm{mg}$ de sélénium. Ces quantités représentaient globalement le double des besoins estimés du dromadaire (14). Le complément minéral était mélangé à de la mélasse, puis à de la farine de riz et distribué individuellement chaque matin. Les auteurs se sont assurés que la totalité du complément était consommée.

3. Une période de post-supplémentation (J113-J195) pendant laquelle tout apport minéral complémentaire a été stoppé. Les animaux ne recevaient donc que la ration de base.

\section{Prélèvements}

\section{Prélèvement de sang}

Les prélèvements de sang étaient réalisés par ponction de la veine jugulaire dans des tubes vacutainer contenant un anticoagulant (héparine ND) non-contaminant en zinc. Après centrifugation, le plasma était collecté avec une pipette et congelé jusqu'à l'analyse. Tous les prélèvements sanguins étaient réalisés le matin, avant la distribution de nourriture et d'eau. Au cours de la première phase de l'essai, les prélèvements ont été effectués au J1, J7 et J21. Douze prélèvements ont été effectués au cours de la seconde phase, soit pratiquement chaque semaine (J24, 31, 38, 48, 55, $62,69,80,87,94,101$ et 108$)$. Le même rythme de prélèvement a été proposé pendant le début de la phase 3 (J115, 122, 129, 136), puis chaque quinzaine jusqu'à la fin de l'expérimentation (J150, $164,178,195)$.

\section{Biopsie hépatique}

Le stockage hépatique étant un important indicateur du statut en éléments-traces, des biopsies du foie ont été réalisées régulièrement afin d'évaluer la dynamique du stockage et du déstockage dans le tissu hépatique. La technique utilisée était une adaptation de celle proposée par Cherrier et coll. (10) chez le chamelon. L'animal était contenu en position de decubitus sternal et recevait un sédatif par voie générale (1 cc de Xylazine, Rompun ND). Le point de ponction, situé sur le côté droit dans le 9 e espace intercostal, à égale distance de la base de la bosse et de l'intersection d'une ligne joignant la pointe de l'épaule à la pointe de la hanche, était lavé et désinfecté avec de l'alcool iodé. Une courte incision $(1 \mathrm{~cm})$ et une anesthésie locale avec de la xylocaïne 2 p. 100 $(5 \mathrm{ml})$ permettaient d'enfoncer une sonde (trocard spécial pour biopsie hépatique) à travers le muscle et le péritoine. La sonde était dirigée vers l'avant. Le tissu hépatique était reconnaissable à sa consistance. A chaque biopsie, 100 à $500 \mathrm{mg}$ de tissu hépatique frais étaient prélevés, puis conservés dans $0,5 \mathrm{ml}$ d'acide sulfurique. Les biopsies étaient suivies d'une désinfection locale et d'une injection intramusculaire (10 cc d'oxytetracycline, TLA ND).

Deux biopsies ont été réalisées au cours de la première phase (J1 et J17), puis chaque mois au cours de la seconde phase (J48, 80, 108) et de la troisième $(\mathrm{J} 136,164,195)$.

\section{Prélèvement de fèces et d'urine}

Pour chaque animal, les matières fécales de $24 \mathrm{~h}$ ont été prélevées, pesées puis séchées (à $100^{\circ} \mathrm{C}$ en étuve) et stockées dans des sacs en plastique en attendant d'être analysées. Le rythme de prélèvement était identique à celui des prélèvements de sang. L'urine de $24 \mathrm{~h}$ a également été collectée selon une méthodologie publiée par ailleurs (5) et selon le même calendrier que les fèces.

\section{Echantillonnage de la ration alimentaire}

Les éléments de la ration de base ont été échantillonnés au début de l'expérimentation, séchés et stockés en attendant d'être analysés. La composition de la ration étant la même tout au long de l'essai, un seul prélèvement a été effectué.

\section{Analyses de laboratoire}

Dans les aliments composant la ration de base, les éléments-traces $(\mathrm{Cu}, \mathrm{Zn}, \mathrm{Fe}, \mathrm{Mn})$ ont été mesurés par spectrophotométrie à absorption atomique selon la technique classique proposée par Bellanger (3). La méthode de Bellanger et Lamand, décrite en 1975 (4) et utilisant également la spectrophotométrie d'absorption atomique, a prévalu pour le dosage des éléments-traces dans le plasma $(\mathrm{Cu}$, $\mathrm{Zn}$ ) et l'urine ( $\mathrm{Cu}, \mathrm{Zn})$.

Les échantillons de tissu hépatique ont été minéralisés par l'utilisation d'acides perchlorique et nitrique selon la méthode préconisée par Bellanger (3). Les échantillons de fèces ont été minéralisés par passage au four à $550^{\circ} \mathrm{C}$ pendant $12 \mathrm{~h}$. Après minéralisation, les échantillons de fèces et de tissu hépatique ont été analysés par spectrophotométrie à absorption atomique. Seuls le cuivre et le zinc ont été recherchés au niveau hépatique. Cette recherche s'est étendue au fer et au manganèse dans l'analyse des fèces.

\section{Méthodes statistiques}

Les analyses de variance ont été réalisées avec le logiciel SYSTAT. Pour chaque variable à expliquer (éléments-traces), les effets de la période de complémentation minérale ( 3 niveaux : avant, pendant, 
après) et du jour du prélèvement ( 23 niveaux pour le sang et les matières fécales ; 8 niveaux pour le foie) ont été testés. La normalité des distributions a été vérifiée au préalable par le test $W$ (test d'asymétrie et d'aplatissement). Les corrélations entre deux variables sont testées par l'estimation du coefficient de corrélation de Pearson.

\section{RESULTATS}

\section{Composition minérale de la ration de base et ingestion des minéraux}

Une partie de la ration de base s'est avérée très carencée en cuivre et en zinc (tableau I). En effet, la paille de blé ne contenait que $2,9 \mathrm{ppm}$ de cuivre et $21,3 \mathrm{ppm}$ de zinc (par rapport à la matière sèche). La teneur en manganèse était particulièrement faible dans la mélasse (4 ppm). Au total, l'ingestion quotidienne de cuivre était de $35 \mathrm{mg}$ pendant les phases 1 et 3 et de $275 \mathrm{mg}$ pendant la phase de complémentation. Ces valeurs étaient respectivement de 145 et de $1145 \mathrm{mg}$ pour le zinc, de 293 et de $1293 \mathrm{mg}$ pour le manganèse. Aucun complément ne contenant du fer, l'apport ferrique a été constant tout le long de l'essai et représentait $330 \mathrm{mg}$ par jour.

\section{Régulation au niveau plasmatique}

La valeur moyenne de la cuprémie était de $61 \mu \mathrm{g} / 100 \mathrm{ml}$ (valeurs extrêmes de 41 à $69 \mu \mathrm{g} / 100 \mathrm{ml}$ ). Au cours des 3 phases de l'essai, la cuprémie moyenne observée était respectivement de 44,63 et $57 \mu \mathrm{g} / 100 \mathrm{ml}$. L'évolution de la cuprémie est illustrée dans la figure 1 et indique un léger effet de la complémentation $(p<0,05)$.

La zincémie observée était nettement plus faible $(38 \mu \mathrm{g} / 100 \mathrm{ml}$ en moyenne avec des valeurs extrêmes de 32 à $45 \mu \mathrm{g} / 100 \mathrm{ml}$ ) et l'évolution au cours des 3 phases de l'cssai (figurc 1) n'indiquait aucun effet notable de la phase de complémentation. Les valeurs moyennes étaient respectivement de 35,36 et $42 \mu \mathrm{g} / 100 \mathrm{ml}$ pour les 3 phases et les différences étaient non significatives.

\section{Stockage hépatique du cuivre et du zinc}

La concentration en cuivre hépatique a varié en moyenne de $10 \mathrm{ppm}$ (par rapport à la MS) pendant la période de contrôle à 26 ppm au cours de la phase de complémentation minérale, puis à $18 \mathrm{ppm}$ pendant la phase de post-complémentation. Pour le zinc, les valeurs étaient respectivement de 43,36 et $45 \mathrm{ppm}$. Comme pour les valeurs plasmatiques, la complémentation minérale n'a eu d'effet significatif que sur la concentration en cuivre. La figure 2 illustre la dynamique de stockage et de déstockage au cours de l'essai.

\section{Tableau I}

Cumpusition de la ration de base en éléments-traces (en ppm)

\begin{tabular}{|c|c|c|c|}
\hline $\begin{array}{l}\text { Elément- } \\
\text { race }\end{array}$ & $\begin{array}{l}\text { Paille } \\
\text { de blé }\end{array}$ & $\begin{array}{l}\text { Farine } \\
\text { de riz }\end{array}$ & Mélasse \\
\hline Curvere & 2,9 & 6,8 & 17,0 \\
\hline zinc: & 21,3 & 53,6 & 14,0 \\
\hline Marganese & 39,1 & 135,5 & 4 \\
\hline Fer & 28,8 & 130 & 68 \\
\hline
\end{tabular}

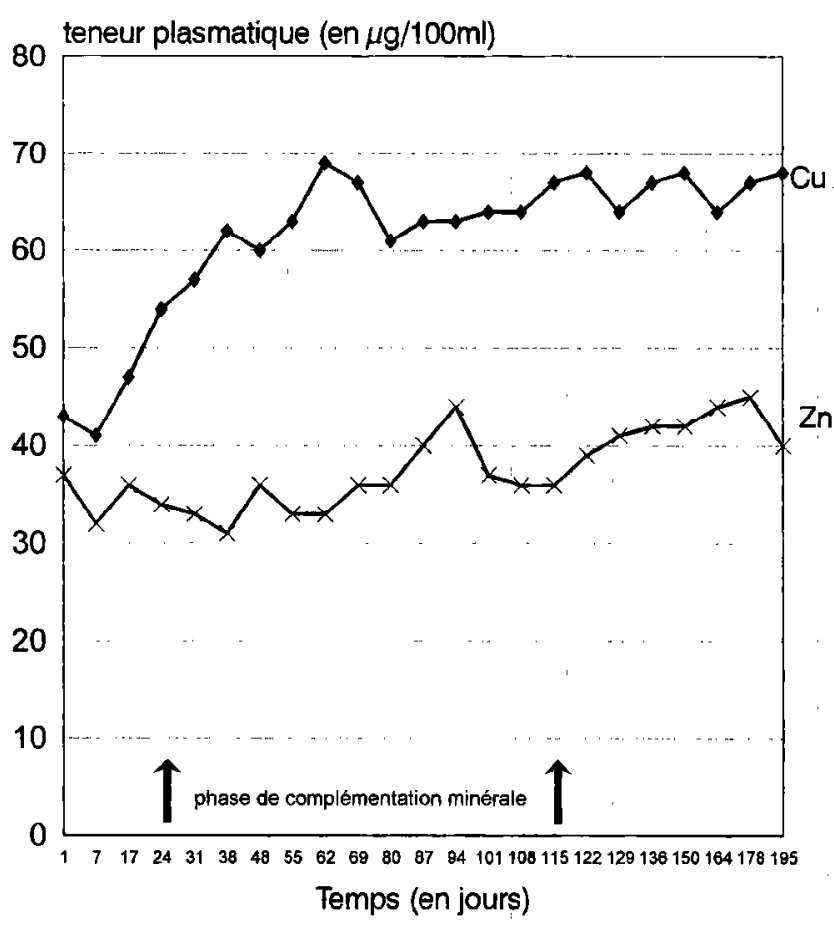

Figure 1 : évolution des valeurs plasmatiques du cuivre et du zinc chez le dromadaire au cours de l'expérimentation. Chaque point représente la moyenne des cinq animaux.

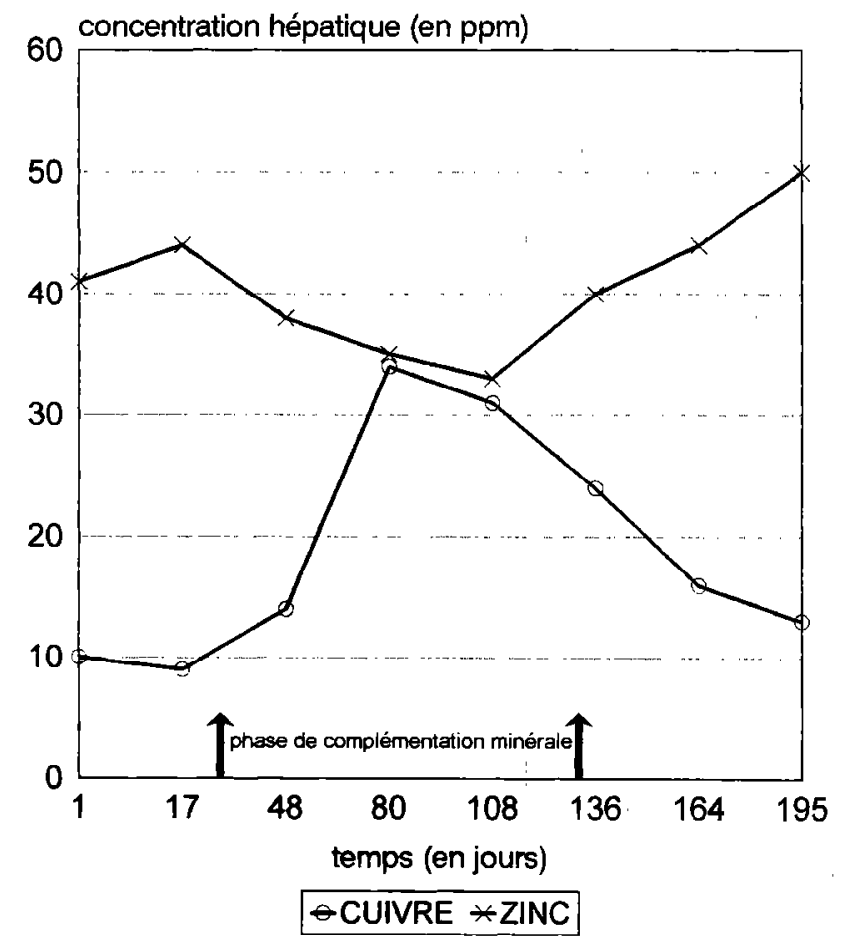

Figure 2 : évolution de la concentration en cuivre et en zinc dans le tissu hépatique du dromadaire. 


\section{Excrétion fécale et urinaire}

La forte augmentation de l'ingestion d'éléments-traces au cours de la phase de complémentation était clairement associée à l'augmentation de la concentration fécale en éléments-traces aussi bien pour le cuivre, le zinc que le manganèse (figure 3). Entre les phases 1 et 2 , l'ingestion de cuivre a été multipliée par 7,8 et l'excrétion fécale par 13,8. Ces valeurs sont respectivement de 4,5 et 7,1 pour le zinc, et de 4,4 et 3,2 pour le manganèse. Concernant le fer, en dépit de l'absence d'apport en sels ferriques, l'excrétion fécale a également augmenté. Elle a été mullipliée par 4,3 et est demeurée élevée pendant la phase 3 (145 $\mathrm{mg} / \mathrm{kg}$ de MS fécales en moyenne).

\section{Absorption apparente}

Les concentrations urinaires en cuivre et zinc sont demeurées anecdotiques, y compris pendant la phase de complémentation. L'urine n'entre donc pas en jeu de façon significative dans les processus d'excrétion. La quantité de MS fécale excrétée quotidiennement n'a, en moyenne, pas changé pendant les deux premières phases, soit $2,2 \mathrm{~kg} / \mathrm{j}$. Le taux d'absorption apparente n'est donc pas influencé par la quantité de matières fécales excrétées. En revanche, cette quantité a nettement diminué pendant la phase 3 , passant à $1,5 \mathrm{~kg} / \mathrm{j}$, ce qui implique qu'en dépit d'un taux d'excrétion sensiblement plus élevé qu'en phase 1, la quantité totale excrétée de minéraux a retrouvé des valeurs comparables à la période précédant la supplémentation, à l'exception du zinc. L'absorption apparente a nettement diminué pendant la phase de complémentation, à l'exception également du zinc dont le rapport cxcrétć/ingéré est demeuré assez faible comparativement aux autres minéraux (figure 4). En moyenne, l'absorption apparente du zinc est passée de 61 p. 100 pendant la phase 1 à 56 p. 100 pendant la phase 2 et 54 p. 100 au cours de la phase d'arrêt de la complémentation. Ces valeurs ont été respectivement de 80,65 et 75 p. 100 pour le cuivre, 78,65 et 81 p. 100 pour le manganèse, et 88,62 et 81,5 p. 100 pour le fer.

\section{DISCUSSION}

A l'exception des travaux déjà anciens de Tartour au Soudan (23, 24), il existe peu d'études faisant le point sur la régulation des minéraux mineurs chez le dromadaire. A la connaissance des auteurs, aucun bilan métabolique (ingestion, stockage, excrétion) n'a été réalisé. Les résultats disponibles dans cette espèce demeurent épars et partiels. Les éléments exposés ici apportent donc des informations nouvelles sur le sujet. La forte augmentation de la digestibilité apparente observée chez les dromadaires lors de la période 3 reste inexpliquée. En effet, la ration est restée inchangée alors que la quantité de matières sèches excrétées a diminué. En l'abscnec d'cxplications ćvidentes, l'interprétation des données de l'excrétion des éléments-traces et des taux d'absorption apparente chez les dromadaires pendant cette période est à considérer avec précaution.

\section{Métabolisme du cuivre}

La complémentation minérale assurait un apport quotidien de l'ordre de $60 \mathrm{mg}$ par $\mathrm{kg}$ de matière sèche, c'est-à-dire un apport largement supérieur à celui généralement préconisé chez des ruminants tels que les bovins (19). En dépit de cet apport jugé considérable, la cuprémie est restée faible, en moyenne à un niveau

\section{$\mathrm{Zn}, \mathrm{Mn}$}

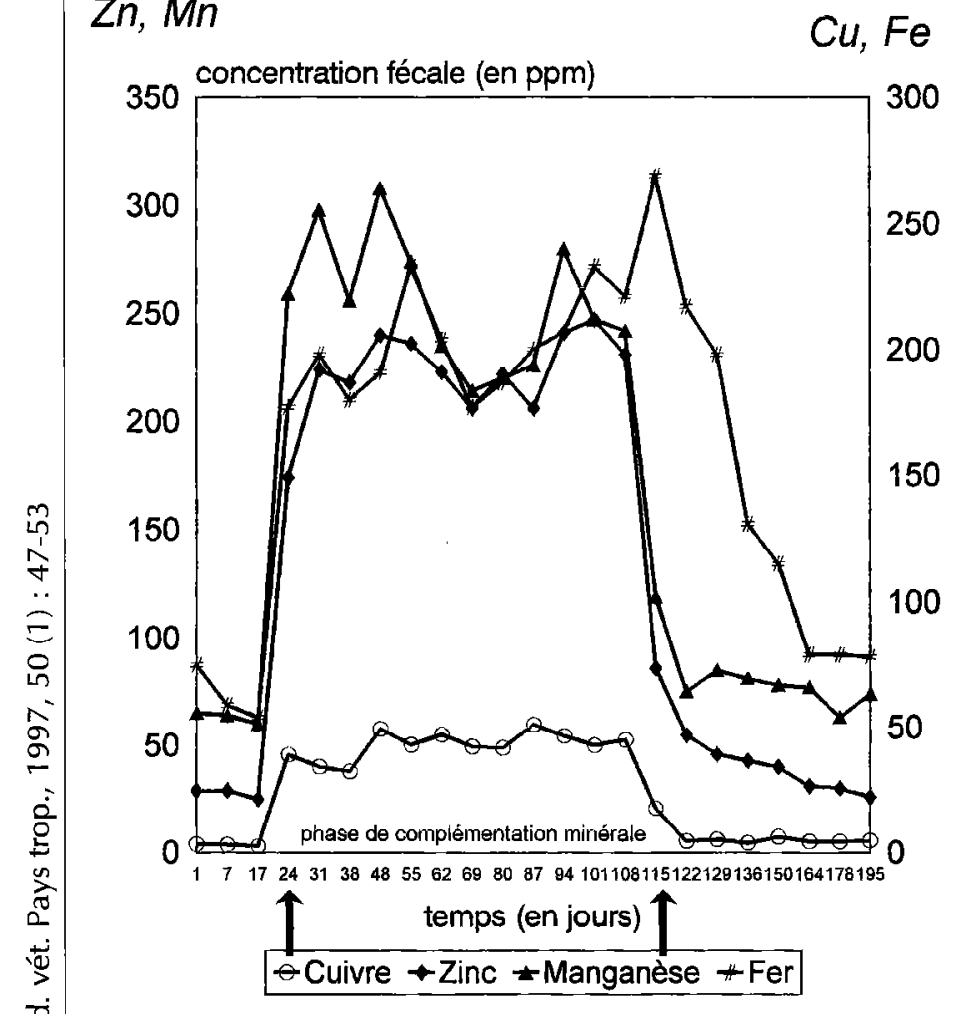

Figure 3 : évolution de la concentration fécale en cuivre, en zinc, en fer et en manganèse chez le dromadaire

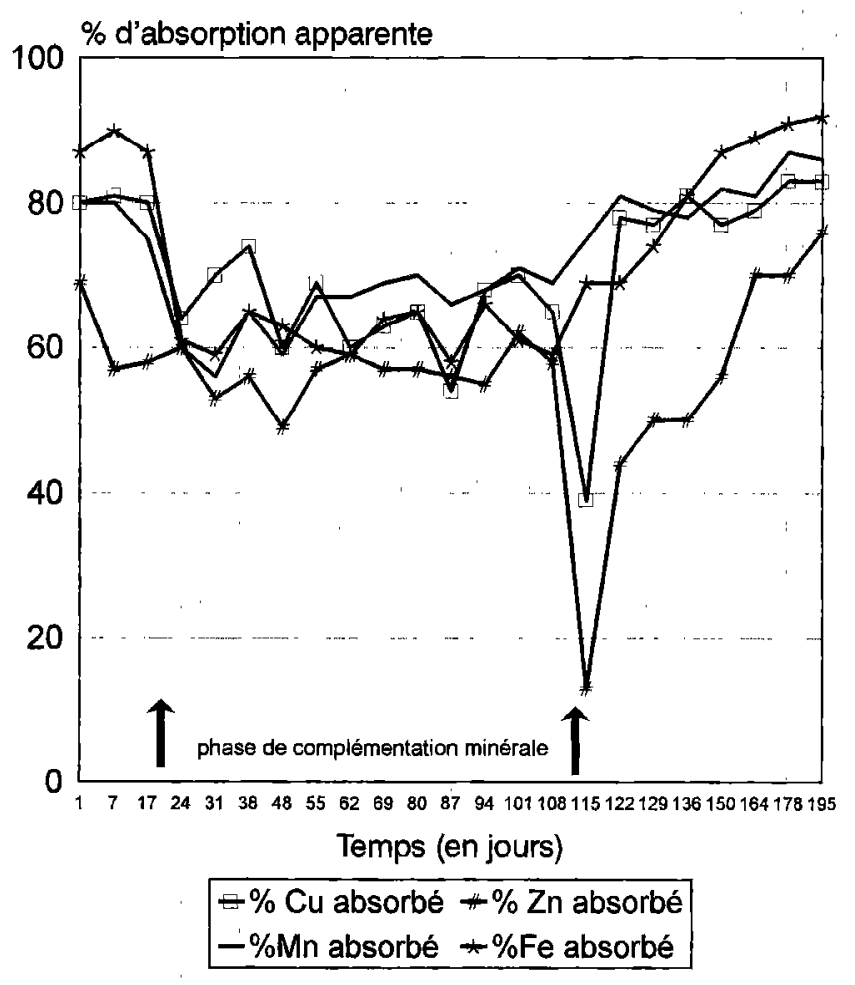

Figure 4 : évolution du taux d'absorption apparente en éléments-traces chez le dromadaire. 
inférieur à la limite de carence préconisée chez les bovins $(70 \mu \mathrm{g} / 100 \mathrm{ml})$ sauf après plusieurs semaines de complémentation. La cuprémie a cependant augmenté significativement pendant la période de complémentation. Néanmoins, elle est demeurée faible par rapport aux résultats de la littérature $(8,11)$. On peut donc suspecter une situation de subcarence au début de cet essai. En effet, la ration de base, n'apportant qu'environ 7,5 ppm de cuivre par $\mathrm{kg}$ de matière sèche, était juste suffisante pour satisfaire les besoins en la matière. En comparaison des bovins, dans des situations d'alimentation analogues, les valeurs de la cuprémie chez les dromadaires sont inférieures (6). Du fait sans doute de la situation de subcarence prévalant au début de cet essai, le stockage hépatique est demeuré peu important et un temps de latence a été observé avant que la concentration hépatique ne se soit améliorée. L'apport complémentaire en cuivre a donc été utilisé en priorité pour rétablir la cuprémie. Inversement, le déstockage hépatique du cuivre a été très rapide en fin de période de complémentation, ce qui a permis un relatif maintien de la cuprémie. Les concentrations en cuivre hépatique sont demeurées faibles par rapport aux bovins (6), mais correspondent aux observations les plus récentes répertoriées chez le dromadaire $(14,26)$.

Cependant, une partie notable du cuivre a été excrétée. Comme pour tous les ions positifs, l'essentiel de l'excrétion se fait par voie fécale. Celle-ci inclut la partie non-absorbée au niveau intestinal et la partie endogène provenant des retours dans la lumière intestinale des minéraux préalablement absorbés. Dans le cas présent, seule la différence entre l'ingéré et l'excrété a pu être mesurée, donnant une estimation grossière de l'absorption apparente. Le pourcentage de cuivre absorbé, de l'ordre de 80 p. 100 en l'absence de complémentation, a diminué sensiblement lors d'apport cuprique. Cette diminution de l'absorption en cuivre pendant la phase de complémentation peut en partie s'expliquer par l'interaction négative cuivre-zinc, celle-ci étant plus importante pour le cuivre que pour le zinc chez le dromadaire (8). Les taux d'absorption chez les dromadaires non-complémentés étaient sensiblement plus faibles que ceux observés chez des bovins (85-86 p. $100 \mathrm{chez}$ les bovins contre $75-80$ p. 100 chez les dromadaires) soumis au même régime alimentaire (6), mais plus élevés en cas de complémentation (65 p. $100 \mathrm{chez}$ les dromadaires contre 61 p. $100 \mathrm{chez}$ les bovins). Ces résultats tendent à montrer que les besoins en cuivre du dromadaire pourraient être moins importants que chez les autres espèces de ruminants. Cependant, en cas de complémentation spécifique, le dromadaire semble présenter une meilleure capacité d'absorption apparente, mettant ainsi à profit un contexte nutritionnel provisoirement favorable. Par ailleurs, le comportement alimentaire du dromadaire en milieu semi-aride (ration plus riche en fourrages ligneux dont beaucoup de légumineuses) lui permet d'accéder plus facilement que les autres ruminants à une alimentation plus riche en azote (22) et en minéraux mineurs (15). Ceci explique, étant donnée l'interaction positive azote-cuivre (14), que le dromadaire «s'en sort » globalement mieux que les bovins, ou même les petits ruminants, dans des conditions naturelles $(12,24)$.

\section{Métabolisme du zinc}

Malgré un apport quotidien de 250 ppm de zinc environ par kg de MS pendant la phase de complémentation, la zincémie observée est restée à des niveaux très faibles, confirmant les résultats récents sur le sujet, aussi bien à Djibouti (14), au Maroc (8) qu'en France (13). Les dromadaires présentent normalement une zincémie faible qui n'est pas influencée par la complémentation minérale. On peut affirmer que la limite de carence se situe plutôt vers $40 \mu \mathrm{g} / 100 \mathrm{ml}$. De plus, la complémentation en zinc ne modifie pas non plus l'état de stock hépatique dont les valeurs observées dans la présente étude sont en général inférieures à celles rapportées dans la littérature $(14,27)$, excepté au Soudan (1). L'excrétion urinaire et pilaire du zinc étant insignifiante, l'excrétion fécale demeure la voie principale d'exportation du zinc d'origine alimentaire et endogène. Or, le rapport excrété/ingéré était de l'ordre de 40 p. 100 pendant la phase 1 et 45 p. 100, en moyenne, pendant les phases 2 et 3 . Le taux d'absorption a donc très peu diminué pendant la phase de complémentation, l'interaction cuivrezinc jouant plutôt en la défaveur du cuivre (8). De plus, contrairement au cuivre, la quantité de zinc excrétée pendant la phase d'arrêt de la complémentation (phase 3) ne s'est pas retrouvée à la hauteur des valeurs de la phase 1 . Il est d'ailleurs remarquable qu'immédiatement après l'arrêt de la complémentation, la quantité de zinc excrétée ait été sensiblement égale à l'ingéré, ce qui ne se produit pas chez les bovins soumis au même régime alimentaire (6). On peut penser que le maintien d'une excrétion fécale élevée après une période de forte complémentation est dû à l'état des stocks endogènes, suffisants pour assurer la demande biologique de l'organisme. Il a fallu attendre un mois après l'arrêt de la complémentation pour observer un rétablissement du taux d'absorption apparente, sans pour autant observer de changement de la zincémie.

L'ensemble de ces résultats permet de considérer que, globalement, les besoins en zinc du dromadaire paraissent faibles. Cependant, ces résultats indiquent aussi qu'il existe un site de stockage extérieur au foie (pas d'augmentation du zinc hépatique) et au système sanguin (peu de changement de la zincémie). L'importante activité enzymatique du rein (5) pourrait conduire à considérer cet organe comme un point potentiel de stockage (20).

\section{Métabolisme du manganèse}

Le dosage du manganèse dans le plasma ou dans le foie ne présente pas d'intérêt clinique (19). Aucun de ces tissus, en effet, ne constitue un reflet fidèle des situations de carence en manganèse. Aussi, cet essai s'est-il limité à l'évaluation de l'ingéré et de l'excrété. En l'absence d'apport minéral, le rapport excrété/ingéré a été de l'ordre de 20 p. 100, en moyenne, et a sensiblement augmenté pendant la phase de complémentation (35 p. 100 en moyenne). Par comparaison avec le cuivre, l'absorpsion apparente a donc diminué pendant la phase de complémentation. Le taux d'absorption apparente en manganèse paraît deux fois plus élevé chez les dromadaires que chez les bovins soumis au même régime alimentaire : 32-48 p. 100 selon la complémentation minérale (6). Il est admis que l'absorption du manganèse est faible chez la plupart des ruminants. Selon certains auteurs, 95 p. 100 environ de cet élément est excrété chez les bovins (18). Le dromadaire présenterait donc une plus grande capacité d'absorption du manganèse que les autres ruminants. En l'absence de stockage hépatique notable $(1,2,27)$ et de stockage rénal (1) ou adipo-musculaire (28), il existe d'autres formes de stockage dans l'organisme, en particulier dans le tissu osseux. On peut considérer qu'à l'instar du zinc les besoins du dromadaire sont globalement couverts par la ration de base, en dépit de la très faible teneur bien connue de la mélasse.

\section{Métabolisme du fer}

Il n'existe pas de carence en fer chez les ruminants pâturant dans des conditions naturelles (26). De ce fait, le dosage du fer plasmatique ne présente pas d'intérêt clinique. Dans les quelques références sur le sujet, le dromadaire présente une sidérémie plutôt plus faible que chez les autres espèces de ruminants $(12,16,25)$. 
Si certains auteurs ont dosé le fer hépatique $(2,27)$, son intérêt demeure limité, l'essentiel du stockage ferrique étant réalisé au niveau sanguin.

Dans cet essai, la concentration de fer fécal (principale voie d'excrétion) a fortement augmenté pendant la phase de complémentation minérale. L'apport alimentaire n'ayant pas changé, cela signifie qu'il y avait une forte interaction avec les autres éléments minéraux apportés par la complémentation minérale. Autrement dit, la complémentation en éléments-traces modifie l'absorption du fer au niveau intestinal. Il existe donc une forte synergie entre les éléments-traces, le cuivre, le zinc ou le manganèse déprimant l'absorption du fer, à l'instar de l'interaction négative observée entre le cuivre et le zinc par compétition de l'absorption au niveau intestinal (8). Toutefois, l'effet déprimant parait moins marqué chez le dromadaire que chez les bovins soumis au même régime alimentaire (6) : le taux d'absorption chez les bovins complémentés n'est que de 50 p. 100 contre 62 p. 100 chez les dromadaires complémentés. Inversement, en l'absence de complémentation minérale, le taux d'absorption ferrique est plus important chez les bovins (environ 90 p. 100) que chez les dromadaires (environ 85 p. 100).

\section{- CONCLUSION}

Le dromadaire a sans doute la capacité d'être moins affecté par les déficits en éléments-traces, ses besoins paraissant moins importants que les autres ruminants en ces nutriments. Ceci est surtout le cas pour le zinc qu'il a la capacité de réguler à des niveaux nettement inférieurs à ceux des autres ruminants. Cependant, le zinc joue un rôle très important dans certaines fonctions enzymatiques en relation avec les capacités immunitaires des animaux, ainsi qu'au niveau de l'intégrité cutanée. Il y a sans doute là des éléments à approfondir, à mettre peut-être en relation avec la forte mortalité des jeunes et la sensibilité de l'espèce aux maladies de la peau. Si les normes des différents éléments-traces chez le dromadaire commencent à être bien décrites, si le métabolisme général devient plus explicite, il reste des points à préciser relatifs aux relations entre les taux circulants et les performances des animaux.

\section{Remerciements}

Ce travail a pu être en grande partie réalisé grâce au soutien de la Fondation internationale pour la science qui a décerné une bourse (B/2293 1) au Dr Bengoumi. Nous remercions également K. Zro, étudiant à l'IAV Hassan II, pour son active participation à la réalisation de cet essai.

\section{BIBLIO GRAPH IE}

1. ABU DAMIR H., TARTOUR G., ADAM S.E.I., 1983. Mineral contents in livestock in Eastern Sudan. Trop. Anim. Health Prod., 15: 15-16.

2. AWAD Y.L., BERSCHNEIDER F., 1977. Values for certain minerals and trace-elements in some tissues of the camel (Camelus dromedarius). Egypt. J. vet. Sci., 14: 31-35.

3. BELLANGER J., 1971. Le dosage des oligo-éléments dans les fourrages. Ann. Nutr. Alim., 25 : 59-96.

4. BELLAN GER J., LAMAND M., 1975. M éthode de dosage du cuivre et du zinc plasmatique. Bull. tech. IN RA Theix, 20 : 53-54.

5. BENGOUMI M., 1992. Biochimie clinique du dromadaire et mécanismes de son adaptation à la déshydratation. Thèse doct. Sciences agronomiques, IAV Hassan II, Rabat, Maroc, $184 \mathrm{p}$.

6. BENGOUMI M., ESSAMADI A.K., TRESSOL J.C., FAYE B., 1996 Comparative study of copper and zinc metabolism on cattle and camel (soumis pour publication).
7. BEN GOU MI M., FAYE B., EL KASMI K., TRESSO L J.C., 1995. Facteurs de variation des indicateurs plasmatiques du statut nutritionnel en oligoéléments chez le dromadaire au Maroc. I. Valeurs usuelles et variations physiologiques. Revue Elev. Méd. vét. Pays trop., 48 : 271-276.

8. BENGOU MI M., FAYE B., TRESSO L J.C., BENGOUMI D., 1995. Facteurs de variation des indicateurs plasmatiques du statut nutritionnel en oligo-éléments chez le dromadaire au Maroc. II. Effet d'une complémentation minérale. Revue Elev. Méd. vét. Pays trop., 48 : 276-280.

9. BENGOUMI M., RIAD F., GIRY I., DE LA FARGE F., SAFWATE A., DAVICCO M.J., BARLET J.P., 1993. Hormonal control of water and sodium in plasma and urine of camels during dehydration and rehydration. Gen. comp. Endocr., 89: 378-386.

10. CHERRIER R., SUMBORO M., FAYE B., 1991. N ote sur une méthode de biopsie hépatique chez le jeune dromadaire. Revue Elev. Méd. vét. Pays trop., 44 : 131-133.

11. FAYE B., BENGOUMI M., 1994. Trace-elements status in camels: a review. Biol. Trace Elem. Res., 41: 1-11.

12. FAYE B., GRILLET C., TESSEM A A., 1986. Teneur en oligo-éléments dans les fourrages et le plasma des ruminants domestiques en Ethiopie. Revue Elev. Méd. vét. Pays trop., 39 : 227- 237.

13. FAYE B., JOUANY J.P., CHACORNAC J.P., RATOVONANAHARY M., 1995. L'élevage des grands camélidés. Analyse des initiatives réalisées en France. INRA Prod. Anim., 8 : 3-17.

14. FAYE B., SAINT-MARTIN G., CHERRIER R., ALI RUFFA M., 1992. The influence of high dietary protein, energy and mineral intake on deficient young camel: II. Changes in mineral status. Comp. Bioch. Physiol., 102A: 417-424.

15. FAYE B., TISSERAND J.L., 1989. Problèmes de la détermination de la valeur alimentaire des fourrages prélevés par le dromadaire. Options méditerr., 2 : 61-65.

16. GHOSAL A.K., DWARAKNATH P.K., JATKAR P.R., 1976. A note on serum iron levels in domestic animals of north-western Rajasthan. Indian J. Anim. Sci., 46: 449.

17. GUEGUEN L., LAMAND M., MESCHY F., 1988. Nutrition minérale. In : Jarrige, éd., Alimentation des bovins, ovins et caprins. Paris, France, INRA, p. 95-111.

18. KHALILI M., LINDGREN E., VARVIKKO T., 1993. A survey of mineral status of soil, feed and cattle in the Selale Ethiopian highlands. II. Trace-elements. Trop. Anim. Health Prod., 25: 193- 201.

19. LAMAND M., 1987. Place du laboratoire dans le diagnostic des carences en oligo-éléments chez les ruminants. Rec. Méd. vét., 163 : 1071-1083.

20. MCDOWELL L.R., 1992. Minerals in animals and human nutrition. San Diego, USA, Academic Press, $237 \mathrm{p}$.

21. RIAD F., BENGOUMI M., GIRY J., DAVICCO M.J., SAFWATE A., BARLET J.P., 1994. Renin-aldostérone axis and arginine-vasopressin responses to sodium depletion in camels. Gen. comp. Endocr., 95: 240-247.

22. RUTAGWENDA T., LECHNER-DOLL M., SCHWARTZ H.J., SCHULTKA W., VON EN GELHARDT W., 1990. Dietary preference and degradability of forage on a semi-aride thornbush savannah indigenous ruminants, camels and donkeys. Anim. Feed Sci. Tech., 35: 179- 192.

23. TARTOUR G., 1966. Study of the role of certain trace-elements in relation to the health of livestock in the Sudan. PhD Thesis, University of London, London, United Kingdom.

24. TARTOUR G., 1975. Copper status in livestock, pasture and soil in Western Sudan. Trop. Anim. Health Prod., 7: 87-94.

25. TARTOUR G., IDRIS O.F., 1970. Studies of copper and iron metabolism in the camel fetus. Acta vet. Brno, 39: 397-403.

26. UNDERW OOD E.J., 1977. Trace-elements in human and animal nutrition, 4th ed. New York, USA, NY Academic Press.

27. WEN SVOORT J., 1992. Copper, iron, manganese and zinc concentrations in livers of race animals. In: Proc. First int. camel symp., Dubai, U nited Arab Emirates, February 2-7, 1991.

28. ZAMIL LE-FAER M., RAWDAH T.N., ATTAR K.M., DAWSON M.V., 1991. Mineral and proximate composition of the meat of the onehumped camel (Camelus dromedarius). Food Chem., 42: 139-143.

Reçu le 25.2.97, accepté le 30.6.97 


\section{Summary}

Faye B., Bengoumi M. New data on the metabolism of main trace-elements in the one-humped camel

In an experiment, a mineral supplementation with copper, zinc, manganese and cobalt sulphates, iodide and selenite had been given for three months, after a three week adaptation period, to five one-humped camels fed with a basal diet covering maintenance requirements. After stopping supplementation, camels were monitored for three more months. Blood, feces samples and liver biopsies were performed regularly to measure copper and zinc levels. Manganese and iron were analyzed in feces and diet only. D uring the three stages of the experiment, mean blood copper levels were 44,63 and $57 \mu \mathrm{g} / 100 \mathrm{ml}$ and mean blood zinc levels 35, 36 and $42 \mu \mathrm{g} / 100 \mathrm{ml}$. Therefore, mineral supplementation increased copper concentrations in plasma and liver, but zinc concentrations did not change. Fecal copper, zinc and manganese concentrations increased during the supplementation stage, but the overall absorption rate decreased from 15 to $20 \%$, except for zinc levels that remained stable. Apparent iron absorption also decreased during the supplementation period, in spite of no iron supplementation, showing a negative interaction between minor minerals and iron. These results suggest that traceelements requirements in one-humped camels are lower than for other ruminants.

Key words: Dromedary - Copper - Zinc - Iron - Manganese Trace-elements - Mineral metabolism - Supplements Morocco.

\section{Resumen}

Faye B., Bengoumi M. Nuevos datos sobre el metabolismo de los principales elementos traza en el dromedario

Durante un ensayo involucrando cinco dromedarios que recibieron una ración de base para cubrir las necesidades de mantenimiento, se distribuyó un complemento mineral a base de sulfatos de cobre, zinc, manganeso y cobalto, de yoduro y selenita, durante tres meses y después de tres semanas de adaptación. Después de la suspención de la suplementación, los animales se siguieron durante otros tres meses. Se tomaron regularmente muestras de sangre, de heces y de hígado por biopsia, para dosificar el cobre y el zinc y para una parte de los sustratos, el hierro y el manganeso. Durante el curso de las tres fases del ensayo, la cupremia media fue de 44,63 y de $57 \mu \mathrm{g} / 100 \mathrm{ml}$ y el nivel de zinc en sangre de 35,36 y $42 \mu \mathrm{g} / 100 \mathrm{ml}$. Por lo tanto, la suplementación mineral aumento las concentraciones de cobre a nivel plasmático y hepático, pero no modificó la tenencia en zinc. La concentración fecal de cobre, zinc y manganeso aumentó durante la fase de suplementación, pero la tasa de absorción disminuyó globalmente de 15 a $20 \%$, excepto la del zinc, que se mantuvo estable. La concentración de hierro fecal aumentó durante la fase de sulementación, a pesar de la ausencia del aporte férrico, indicando una interacción negativa entre los minerales menores y el hierro. El conjunto de los resultados lleva a considerar que las necesidades del dromedario en elementos traza son más bajos que para los otros rumiantes.

Palabras clave: Dromedario - Cobre - Cinc - Hierro Manganeso - Oligoelemento - Metabolismo de minerales Suplemento - Marruecos. 